\title{
Intrinsic Bio-Enhancer Entities of Fagonia cretica for Synthesis of Silver Nanoparticles Involves Anti-Urease, Anti-Oxidant and Anti-Tyosinase Activity
}

\author{
Aqsa Yousaf ${ }^{*}$, Ayesha Zafar ${ }^{*}$, Muhammad Ali2², Snober Mona Bukhary3, Yasmeen Manzoor1, \\ Tuba Tariq1, Asma Saeed ${ }^{2}$, Muhammad Akram¹, Faryal Bukhari', Muhammad Abdullah", \\ Syeda Sadaf Zehra5 ${ }^{5}$, Shahbaz Gul Hassan6", Murtaza Hasan"\# \\ ${ }^{1}$ Department of Biochemistry and Biotechnology, The Islamia University, Bahawalpur, Pakistan \\ ${ }^{2}$ Department of Biological Sciences, Gomal University, Dera Ismail Khan, Pakistan \\ ${ }^{3}$ Department of Chemistry, The Islamia University, Bahawalpur, Pakistan \\ ${ }^{4}$ Cholistan Institute of Desert Studies (CIDS), The Islamia University, Bahawalpur, Pakistan \\ ${ }^{5}$ Department of Botany, The Islamia University, Bahawalpur, Pakistan \\ ${ }^{6}$ College of Information Science and Engineering, Zhongkai University of Agriculture and Engineering, Guangzhou, China \\ Email: "mhasan387@cau.edu.cn, "murtaza@iub.edu.pk
}

How to cite this paper: Yousaf, A., Zafar, A., Ali, M., Bukhary, S.M., Manzoor, Y., Tariq, T., Saeed, A., Akram, M., Bukhari, F., Abdullah, M., Zehra, S.S., Hassan, S.G. and Hasan, M. (2019) Intrinsic Bio-Enhancer Entities of Fagonia cretica for Synthesis of Silver Nanoparticles Involves Anti-Urease, Anti-Oxidant and Anti-Tyosinase Activity. Advances in Bioscience and Biotechnology, 10, 455-468.

https://doi.org/10.4236/abb.2019.1012032

Received: September 4, 2019

Accepted: December 22, 2019

Published: December 25, 2019

Copyright $\odot 2019$ by author(s) and Scientific Research Publishing Inc. This work is licensed under the Creative Commons Attribution International License (CC BY 4.0).

http://creativecommons.org/licenses/by/4.0/

(c) (i) Open Access

\begin{abstract}
Objective: Evaluating the bio-reducing activity and various potential, annotates the biological entitties of Fagonia cretica. Method: By fractionating the crude extract with different ethanol-water fraction mixture. This substantiates the reduction potential for the biological synthesis of AgNPs, its antioxidant, anti-urease and anti-Tyrosinase activity. Results: UV-visible spectra confirm the bio-reducing potential for Ag NPs with the optimized 50\% ethanol-water fraction mixture. Transmission electron microscopy (TEM) confirmed the round shaped AgNPs with an average size of $16 \mathrm{~nm}$ in optimized 50\% fraction mixture. Further evaluating Ethanol-water fraction with $90 \%$ and $70 \%$ of plant extract showed more potentials for Anti-Tyrosinase and Anti-urease activity. Moreover, diluted 30\% fraction exhibits two folds higher Anti-oxidant and Anti urease activity. Conclusion: Overall, this work showed that 50\% Ethanol-water fraction has potentially active molecules (among all fractions) primarily involved in bio-reducing potential of Ag NPs and enzymatic assay while $90 \%$ mixture proved to be least active. This study suggested electing the optimum mixture fraction of $50 \%$ ethanol-water in order to screen the bioactive compounds and to inculcate their activity status for clinical trials.
\end{abstract}

*These authors contributed equally to this work. 


\section{Keywords}

Bioactivity, Fagonia cretica, Nanoparticles, Enzymes Assay, Bio Reducing Agent

\section{Introduction}

Green synthesis of nanoparticles is current and most fascinating research currently on going in the field of Nano biotechnology [1] [2] [3] [4]. Advances show that many plants have bio-reducing potential for the synthesis and to gain stability of particles to enhance their bio-medical application [5] [6] [7], which exhibits a remarkable degree of complexity needs to be integrated by making fractions for further verifications of bioactive components. Plants being a traditional means of synthesizing medicine for prevention and cure of diseases, food industry for maintaining and preserving the quality of food and in the field of cosmetic, provides with natural and harmless bioactive components [8] [9] [10] [11] [12]. Fagonia cretica is a forested small thorny herb, fit into family Zygophyllaceae, belongs to the chief group of flowering plant Angiosperm. This plant spread in to barren and hot areas of the world, mostly seen in dried out rocks (calcareous) throughout Pakistan and all other continents excluding Australia [13]. In Pakistan, it is generally known as Dhamasa [9]. Medicinal importance has increased the demand as it is anti pyretic and preventive in contrast to small pox [14]. Boiled plant of Fagonia cretica is used as a cure for hepatitis Sheddried plant of Fagonia cretica is important for gynaecological problem [15] [16]. Juice of crushed leaves of Fagonia plays important role in purification of blood, used against gas problems and worm's infections [17]. It can be applied as a paste on external side of tumours. Thus leaf instillations are used in the treatment of cough, asthma problems, edema, act as flatulence reliever and constringent [18]. Antioxidant potential of Fagonia extract was recently reported against the Chemical Ischemia-Induced in PC12 Cells [19]. In another reports, antioxidant potential of Fagonia different parts extras was tested in vitro and made comparison [20] [21]. Anti-urase and anti-tyrosinase activity of Fagonia plant is not yet reported in extract as well as fraction form.

Clear-sighted complexities compelled to elaborate the use of plant in dried form, pure, aqueous, methanolic, alcoholic with their combination of aqueous medium for ethnomedical studies including antioxidant, hematology, Antiradiation, diabetes, anticancer activities and others using in vitro and in vivo analysis [22]-[29]. Each prepared extract provided district response towards certain specific activity. Simply as methanolic extract exhibited significant antimicrobial potential chiefly. But previously, much contribution is done to determine the biomedical application of the extract.

Thus selection, combination and then fractionation of the solvents are our matter of concern to address the exact bioactive compound. So far no interrogation is done on making fraction mixture with suitable solvent to analyze the 
bioactivity with accuracy of whole entire plant. Now here we propose to prepare fractionation mixture of ethanol and water, sub categorized into four different percentages such as $90 \%, 70 \%, 50 \%$ and $30 \%$. For this pure Fagonia cretica extract was prepared using fraction mixture with ethanol and water with different ratio was carried using fractionation to evaluate its bioactivities such as anti-tyrosinase, anti-urease, antioxidant and bioreducing potentials for synthesis of silver nanoparticles for the first time. That function as the measure of potentials residing in the plant extract against the oxidants provided with different dilutions fractions to reduce the complexity. Antiurease, antityrosinase, and antioxidant activity of Fagonia cretica are thus evaluated, further elucidating the bio-reducing potential imparted in the synthesis of silver nanoparticles for all designed fractions.

\section{Materials and Methods}

\subsection{Chemical and Reagents}

All chemicals used were of analytical reagent grade. Sodium Nitroprusside, Ascorbic acid, Glacial acetic acid and Tripyridyl-striazine (TPTZ), Substrate L-Tyrosine, Kojic acid, Phosphate Monohydrate, Urea, Urease, Thiourea, Alkali reagent, Phenol reagent, $\mathrm{AgNO}_{3}$. 2,2-diphenyl-1-picrylhydrazyl (DPPH).

\subsection{Preparation of Plant Extract}

Plant of Fagonia cretica were taken from CIDS Department (Cholistan Institute of Desert studies) at The Islamia University of Bahawalpur, Pakistan. Fresh plant material was washed with distilled $\mathrm{H}_{2} \mathrm{O}$, cut into tiny parts and kept below shade until it was dried. Whole plant was shed dried and crushed using pestle \& mortar to obtain its powdered form. Later on $20 \mathrm{~g}$ of extract powder was dipped in mixture of ethanol and water with $90 \%$ and $10 \%$ dilution respectively. After overnight incubation filtration was done and the filtrate was dried and prepare powder as fraction (90\%) for further used. Than same procedure was repeated with ethanol and having $70 \%$ and $30 \%$ dilution respectively and then same process was repeated twice to get $50 \%$ and $30 \%$ ethanol dilution of plant extract with the help of ethanol and water. Fraction of $90 \%, 70 \%, 50 \%$ and $30 \%$ ethanol dilution were kept in refrigerator at $4^{\circ} \mathrm{C}$ temperature for further activities.

\subsection{Bio-Reducing Activity via AgNPs Synthesis}

For the synthesis of nanoparticles, 0.1 molar solution of $\mathrm{AgNO}_{3}$ was prepared. Reagent bottle contains $25 \mathrm{ml}$ of $\mathrm{AgNO}_{3}$ with $5 \mathrm{ml}$ of different plant extract (90\%, 70\%, 50\% and 30\%) to make final volume $30 \mathrm{ml}$. This mixture was put on magnetic stirrer $(150 \mathrm{rpm})$ and let it there for $5 \mathrm{hrs}$. at $50^{\circ} \mathrm{C}$ as reaction is carried out. There was change in color that is the indication of nanoparticles synthesis. After it, mixture was kept at centrifugation at $6000 \mathrm{rpm}$ for 20 minutes. After changing the color, mixture was used to do the Uv-Vis for confirmation of Ag Nanoparticles synthesis. Transmission electron microscopy (TEM) images of syn- 
thesized AgNPs with the help of all fractions were taken from JEOL JEM 2100.

\subsection{Antiurease Activity}

Antiurease activity of Fagonia cretica fractions were performed. Urease enzyme solution $20 \mu \mathrm{l}$ of mixed with $55 \mu$ of phosphate buffer $(0.2 \mathrm{M})$ having PH 7.4. The mixture was kept on incubation for 10 minutes at $3^{\circ} \mathrm{C}$. Later, add $15 \mu \mathrm{l}$ urea and kept it again for incubation at the same time and temperature. After incubation known volume of Fagonia each extract $(10 \mu \mathrm{l})$ were added in 96 wells that is followed by addition of $10 \mu \mathrm{l}$ of Thiourea (standard solution). After proper mixing again kept at incubation for 10 minutes at $37^{\circ} \mathrm{C}$. Then add $40 \mu$ l Alkali reagent and $60 \mu \mathrm{l}$ phenol reagent in all wells and leave it at room temperature for 10 minutes. Absorbance was measured spectrophotometric at $625 \mathrm{~nm}$. Urease inhibition percentage was calculated by using mentioned formula

$$
\% \text { inhibition }=\left[1-\left(\mathrm{A}_{625} \text { of sample } / \mathrm{A}_{625} \text { of control }\right) \times 100\right] .
$$

\subsection{Anti-Tyrosinase Activity}

To check the potential of Fagonia cretica against melanogenesis, anti-tyrosinase activity was performed. The reaction mixture in 96 well plate contained $140 \mu \mathrm{l}$ of phosphate buffer having $\mathrm{pH} 6.8$ with or without samples with different concentration $(5 \mu \mathrm{l}, 10 \mu \mathrm{l}, 15 \mu \mathrm{l})$ and l-DOPA $(0.5 \mathrm{mM})$. were added in wells. Kojic acid was used as (standard solution). After proper mixing kept plate at incubator for 15 minute at $37^{\circ} \mathrm{C}$. Later L-tyrosine was added in all wells. Mixture was again incubated for 15 minutes at $37^{\circ} \mathrm{C}$. Quantity of Dopachrome (O-dopaquinone) was measured spectrophotometrically at $475 \mathrm{~nm}$. Tyrosinase inhibition percentage was calculated by using mentioned formula [30].

$\%$ inhibition $=\left[\left(\mathrm{A}_{475}\right.\right.$ of control $-\mathrm{A}_{475}$ of sample $) / \mathrm{A} 475$ of control $] \times 100$.

\subsection{Anti-0xidant Activity}

To determine anti-oxidant potential of Fagonia cretica, NO radical scavenging assay were used. NO is biologically functional but its chemistry is very complicated. Sodium phosphate buffer of known volume $(175 \mu \mathrm{l})$ and PH (7.4) was added in required wells of 96 wells plate that was followed by $20 \mu$ of Sodium Nitroprusside and $5 \mu \mathrm{l}$ of sample in all wells. Properly blended all the contents and incubated for 3 hours at $37^{\circ} \mathrm{C}$ before adding Griess reagent. After incubation $20 \mu \mathrm{l}$ of Griess reagent was added in each well. Further incubation was done for one hour (at room temperature. Absorbance was measured spectrophotometer at $528 \mathrm{~nm}$. Analysis of all samples was done for triple times. Nitric oxide scavenging activity was measured by using following formula.

$$
\begin{gathered}
\% \mathrm{NO} \text { scavenging activity } \\
=\left[\left(\mathrm{A}_{528} \text { of control }-\mathrm{A}_{528} \text { of sample }\right) / \mathrm{A}_{528} \text { of control }\right] \times 100 .
\end{gathered}
$$

\section{Results and Discussion}

Synthesizing silver nanoparticle and monitoring the extraction yield of extracta- 
ble bio-entities from Fagonia cretica using ethanol and its subsequent fraction helped in providing a reliable and probative method for successful synthesizing Ag NPs with all fraction.

By correlating the separated and resolved bioactive components on the bases of their chemical nature and solubility to the bio-reducing potential Ag NPs. Potentials such as, anti-oxidant, anti-Tyrosinase, and anti-urease residing inside the plant become superficially expressive that enhances the bio-reducing potential by modulating the ethanol concentration and latterly making its dilutions. Due to different chemical nature of biomolecules, the study aims to biologically synthesize Ag NPs by provide chemically suitable environment for those bioactive components responsible for the synthesis, to deliver their maximum activity by making four different sets of ethanoic concentration in Figure 1. That provided, quite significant results. But in order to optimize and confirm the bioactivities that are being manipulated by bioactive entities accountable for the green synthesis, two parallel series of dilutions were carried out as dilution 1 (D1) and dilution 2 (D2). That reveals the masked activity, on simplifying the complexities by making dilutions and finally confirming the bio-reducing potential of synthesized Ag NPs.

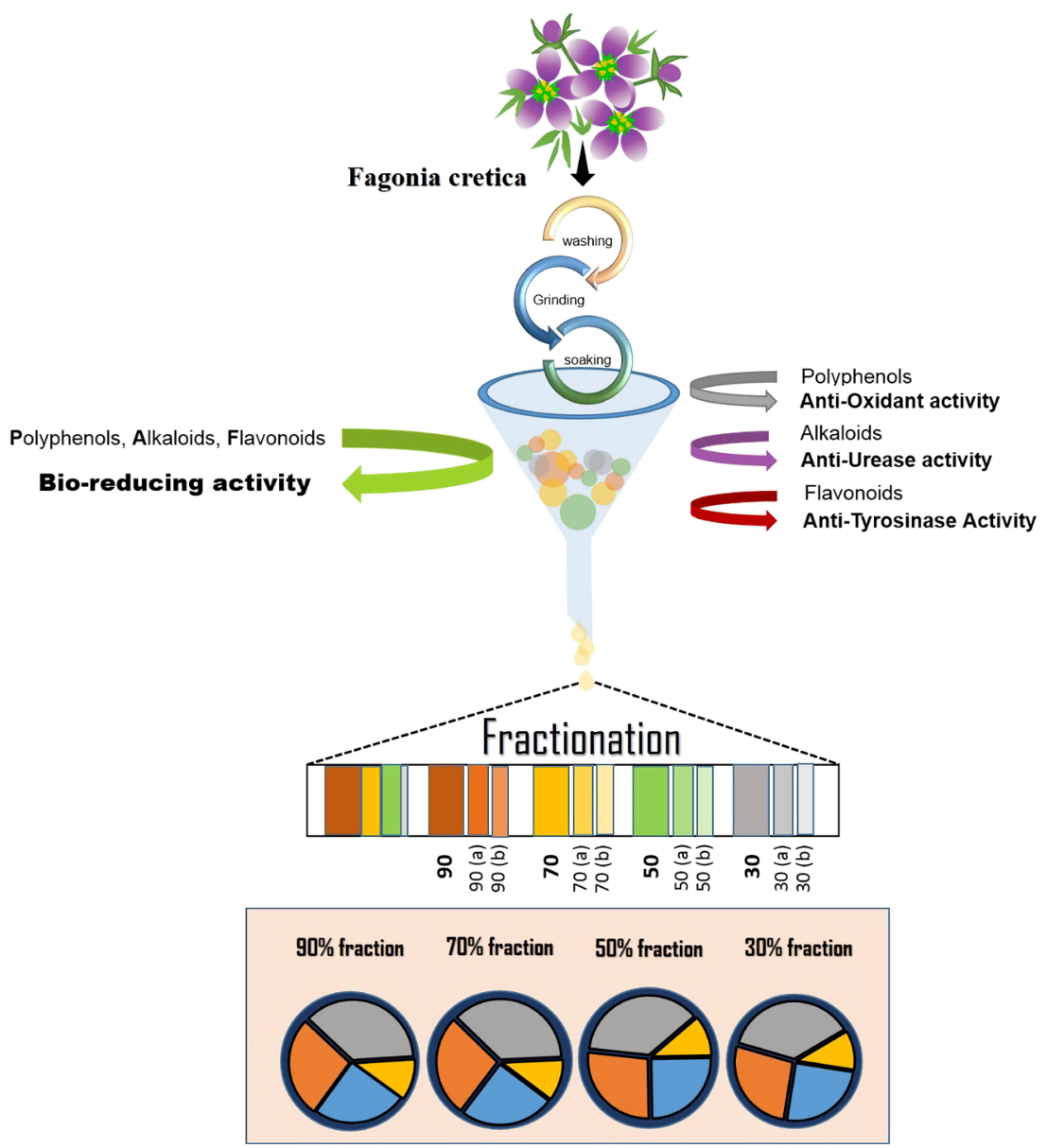

Figure 1. Schematic showing the fractionation of Fagonia cretica, their spectra and bioactivity. 


\subsection{Bio-Reducing Activity for AgNPs Synthesis}

Owing the bio-reducing potential illustrated in Figure 2. This has shown that pure $50 \%$ fraction mixture was the best fraction in synthesizing the NP. Whereas $90 \%$ and $70 \%$ plays comparatively less role in NPs synthesis. But the pure $30 \%$ fraction mixture was proven insignificant.

Bio reduction process of silver nanoparticles formation usually shows absorption peak from 390 - $450 \mathrm{~nm}$ done by UV visible spectroscopy. Solution of silver nitrate without plant extract was used as a control. Several biomolecules of plants enzymes, alkaloids, polysaccharides, amino acid, alcoholic compounds, vitamins and proteins) may cause bio reduction and synthesis of silver nanoparticles [2]. On exposure to plant extract silver ions reduces to silver nanoparticles followed by change in colour and UV visible spectroscopy. From Figure 2, it had been shown that 50\% Fagonia fractions gives maximum peak, while 90\%, 70\% and $30 \%$ fractions had shown much reduced peak having no bio reduction potential.

Further confirming the size and morphology of the synthesized Ag NPs transmission electron microscopy (TEM) is used with different mixture fraction of ethanol in Figure 3. Which clearly depicts round shaped nanoparticles with an average size of $16 \mathrm{~nm}$, using $50 \%$ of fraction mixture, that provided with the most significant results among all fractions as shown in Figure 3(c). 90\% and $70 \%$ also accounts for the synthesis but was less significant as in Figure 3(a), Figure 3(b). While Figure 3(d) exhibits 30\% fraction mixture that gave non-significant results. This is because of the specific components that is showing its maximum activity with certain fraction mixture. In this case $50 \%$ fraction mixture provides optimized ration of maximum bioactive entities, exhibiting their maximum potential on average and thus optimising the bio reducing potential of synthesized Ag NPs. In other fractions there is considerable alteration in the anti-oxidant, anti-Tyrosinase, and anti-urease, where one gets activated

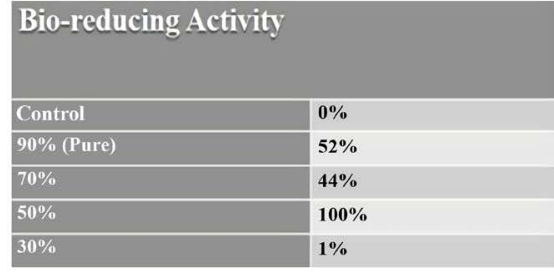

Bio-Reduction

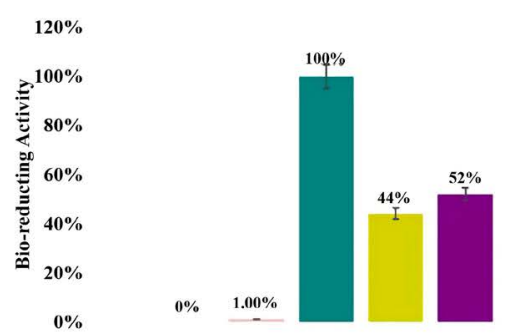

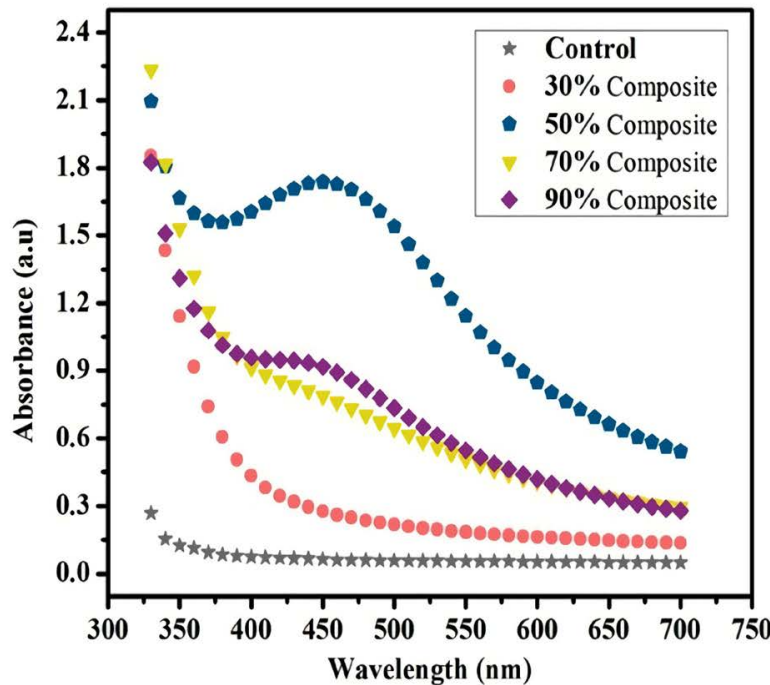

Wavelength $(\mathrm{nm})$

Figure 2. Bioreducing Activity of Fagonia cretica Fraction for synthesis of silver nanoparticles. 

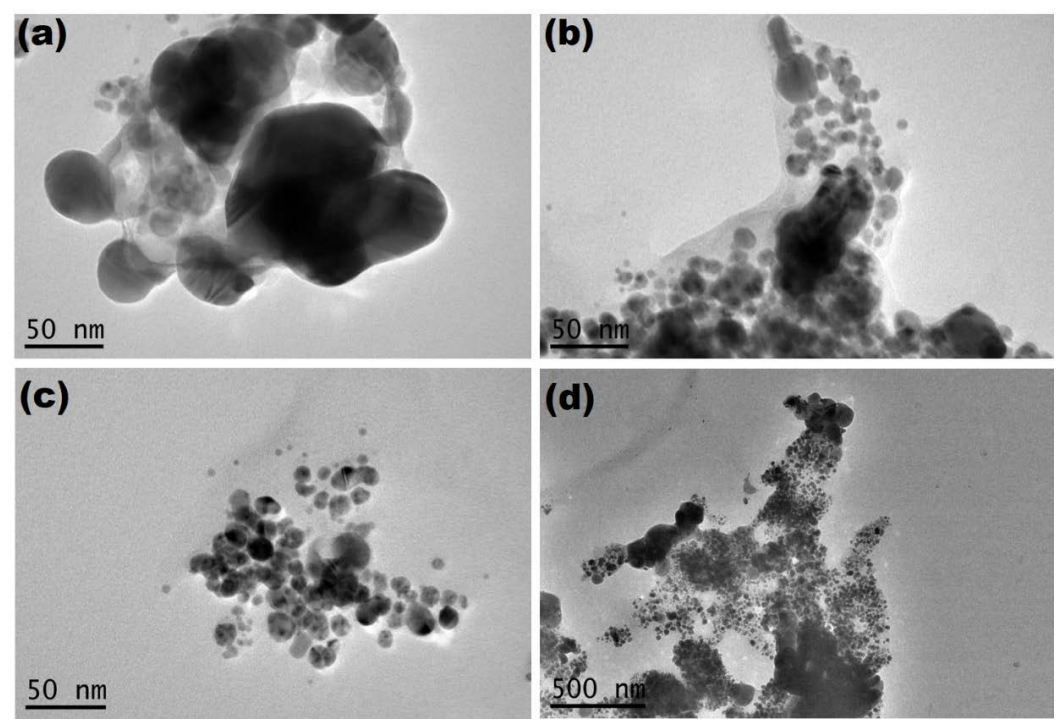

Figure 3. TEM imaging of Fagonia cretica with different Fraction of synthesis of Ag Nanoparticles.

and other gets supressed in its pure and dilute form, effecting the bio reducing potential on the whole for Ag NPs synthesis.

\subsection{Anti-Urease Activity}

The resultant Anti-Urease Activity (AUA) of the pure extract along with dilutions are enlisted in Figure 4. Herein comparing the control Thiourea providing $77 \%$ of AUA, with $90 \%$ fraction mixture shows up to $87 \%$ activity with pure, $93 \%$ activity with D1, and about $64 \%$ activity with D2. $70 \%$ fraction mixture gave $77 \%>52 \%>50 \%$ with pure $>\mathrm{D} 1>\mathrm{D} 2$ respectively. Next for $50 \%$ fraction mixture AUA is $67 \%$ with the pure, but negative results with the D1 and D2 were observed with the control. However, this fraction was considered to exhibiting more significant results regarding other bioactivities in plants. Followed by the next $30 \%$ fraction exhibited $60 \%<82 \%<106 \%$ with the pure $<\mathrm{D} 1<\mathrm{D} 2$ showing a remarkable AUA. Conclusively $90 \%$ and $30 \%$ fraction mixture and its dilution had shown considerably high AUA.

As alkalinity in biological system is produced due to conversion of urea to ammonia by urease, that invites the bacteria to grow and causing severe infection [31]. Urease blocking agents are currently under consideration for assessment of ulcer and other infections which are produced by urease releasing bacteria, etc. Therefore, new treatment methodologies are established focusing on inhibition of urease activity for the cure of infectious diseases. Present work reports that, In the qualitative analysis of all the Fagonia fractions (50\%, 60\%, 70\% and $90 \%$ ) displayed some gradation of qualitative inhibition expressed by changing in color bands from light blue to dark blue which indicates that the fractions contain some active biomolecules capable of binding to the enzyme and producing a significant color change and avoid hydrolysis of urea. Actually urea reacts with reagent to give blue coloured complex. If plant extract has Urease 

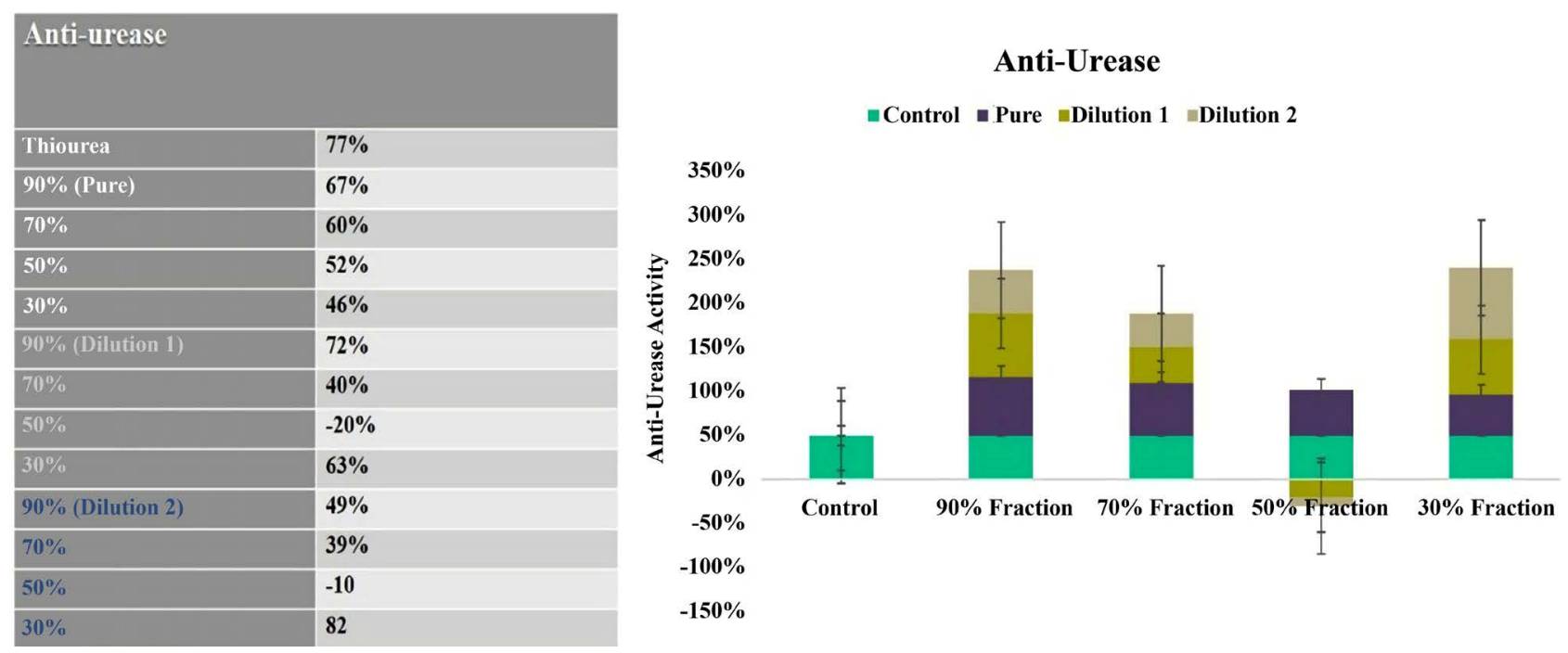

Figure 4. Anti-Urease activity of Fagonia cretica pure fraction and its dilution.

inhibition potential than blue color of the assay reduces. It had also been observed that pure fraction $90 \%$ and dilution I had shown maximum anti-urease potential with respect to the control (Thiourea) ensuring the presence of bio-active molecules. Antiurease assay shows that 1 st two wells of plate contain the control that gave no color change. The color of wells from $3^{\text {rd }}$ to $6^{\text {th }}$ gradually change from light blue to dark blue loaded with mixture fraction $90 \%$ to $30 \%$ respectively as compared to the control, exhibiting the reduction of anti-urease activity. It was further investigated that Fagonia with fraction $90 \%$ dilution I and fraction 30\% with dilution I and dilution II had shown significant activity against the urease. Previously Armando et al. reported that urease inhibitory activity of methanol extracts from ten Guatemalan Piper species [32]. Here, all fractions demonstrated significant correlation regarding anti-urease activity in comparison with the control, which conformed the already published data [33].

\subsection{Anti-Tyrosinase Activity}

Anti-Tyrosinase activity assessment are listed in Figure 5. Keeping Koijic acid as control, $90 \%$ fraction mixture showed $93 \%<125 \%<140 \%$ activity, $70 \%$ fraction mixture had shown activity as $100 \%>66 \%<90 \%$, next in $50 \%$ fraction, where the activity was $26 \%<52 \%<96 \%$ and the last $30 \%$ prepared fraction mixture contributed $73 \%<85 \%<106 \%$ of the total anti-Tyrosinase activity with the pure $<$ D1 < D2 with respect to the set control. Resultantly, the $90 \%$ and $30 \%$ fraction mixture with dilutions panoramic the anti-Tyrosinase activity that was much higher than the control, ensuring the tremendous potential of Fogonia Cretica against Tyrosinase activity.

Tyrosine based peptides or biomolecules have potential to show tyrosinase activity via oxidation of L-3, 4-dihydroxyphenylalanine. It leads to the decomposition of melanin (pigment) thereby facilitating whitening of skin [34]. The expected natural products present, includes phytochelatin peptides, flavonoids, 

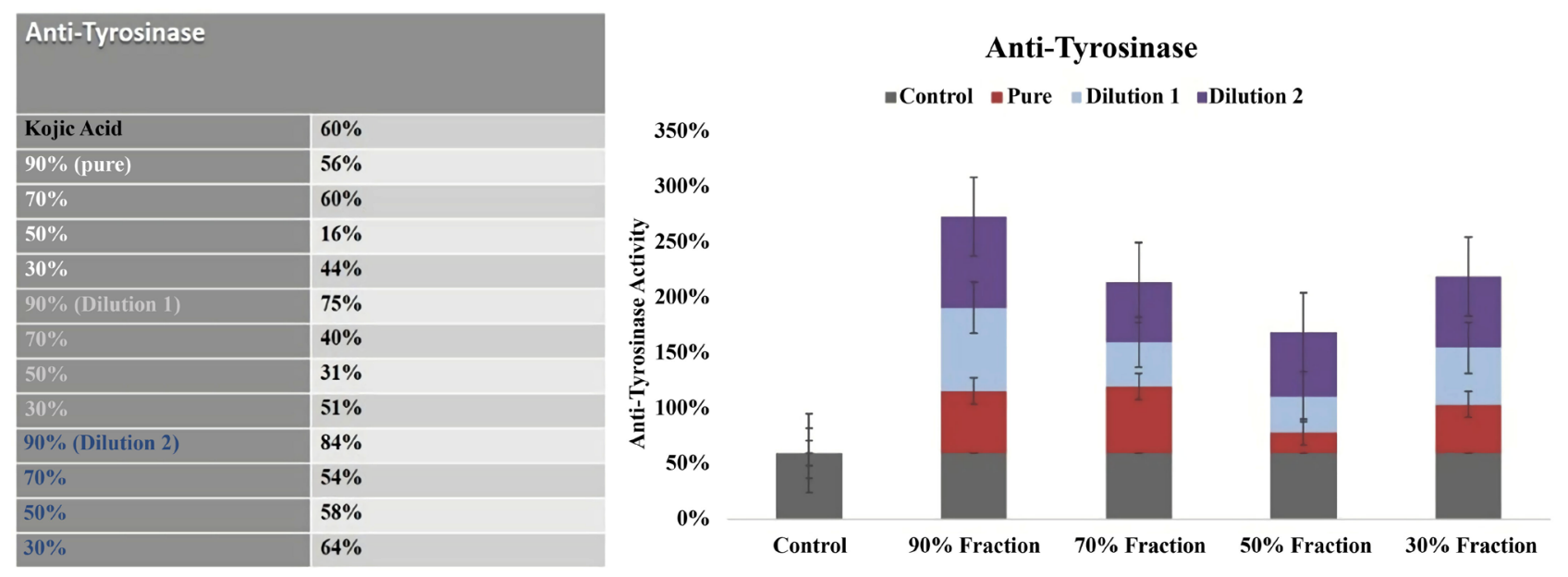

Figure 5. Inhibition of Tyrosinase activity by Different Fractionation of Fagonia cretica.

aromatic acids, polyphenols, or aromatic aldehydes as these compounds can act as effective competitive inhibitors of melanin synthesis of melanin [35]. Variation in the activity of mushroom tyrosinase can be attributed to the presence of tannins in the extracts are capable precipitate proteins thereby limiting the tyrosinase inhibition [36].

From Figure 5, the screening data exhibited that $90 \%$ and $30 \%$ fraction of Fagonia shows strong inhibition potential among all its dilutions but $50 \%$ dilution shows lowest percentage inhibition as compared to standard kojic acid. In $1^{\text {st }}$ two well had shown anti-tyrosinase assay of negative control. That confirms the formation of dopachrome. In $2^{\text {nd }}$ row, no color change was observed due to inhibition of Dopachrome formation. In these wells standard inhibitor was kojic acid. $3^{\text {rd }}$ to $6^{\text {th }}$ line shows antityrosinase potential of different dilutions of Fagonia cretica at different concentrations. Inaddition, it has been also observed that dilution I and Dilution II of $90 \%$ fraction of Fagonia had also shown a significant difference in their activity as compared to all other fraction dilutions. And amazingly higher anti-tyrosinase potential than that of control. Thus proving Fagonia cretica as very strong candidate that exhibits high potential against tyrosinase. The separation of multiple bio-active compounds and along with their structural explanation will provide a chance to develop more potent skin whitening and anti-aging products.

\subsection{Anti-Oxidant Activity}

Anti-oxidant activity investigation is shown in Figure 6. Results and calculations had revealed that it provides negative or null effect with the pure fraction mixture except, pure $50 \%$ that is $7 \%$ more active than the control. In D1 only $50 \%$ and $30 \%$ fraction mixture provided $103 \%$ and $75 \%$ activity. Where pure $50 \%$ is considered as good-dilution fraction showing activity $3 \%$ higher than that of control. Next in the D2, 90\% > 70\% > 50\% > 30\% fraction mixture gave $25<$ $107 \%>50 \%<111 \%$ of activity with respect to control respectively. Depending upon the fraction mixture $50 \%$ fraction mixture were proved nearly significant 


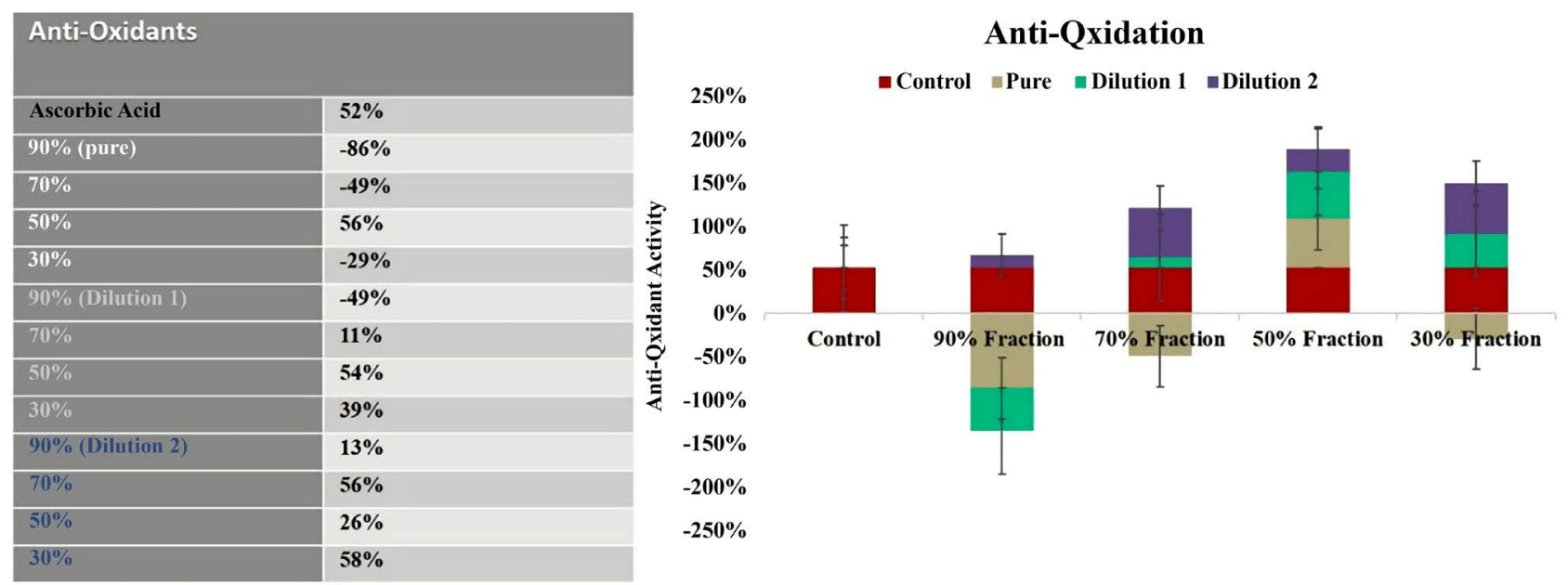

Figure 6. Fagonia cretica fraction and their Antioxidant with dilution.

results in its pure and D1 but overall D2 set of mixture gave far more significant results that expected with the control.

Antioxidant potential of Fagonia cretica removes potentially harmful NO. Garrat method of NO scavenging radical was used. According to this method, there should be reduction of $\mathrm{NO}_{3}^{-}$into $\mathrm{NO}_{2}^{-}$in the sample. Nitrite ions quantification was done by Griess reaction. Pink color complex is generated at the end of reaction with Vitamin $\mathrm{C}$ set as control. Plant extract with antioxidant activity cause reduction of pink color. It is concluded that Fagonia fraction 90\%, $70 \%$ and $30 \%$ extract had lower radical scavenging or antioxidant activity as it gave dark pink color, whereas $50 \%$ fraction mixture gave a colour change to light pink color that assured a significant antioxidant potential. Previous studies also demonstrated that methanolic extracts of roots and aerial parts of Fagonia cretica has anti-oxidant potential against oxidative stress [16]. This reveals that this plant may be used as a cure for oxidative stress mediated disorders. Surprisingly more significant results had been observed with the prepared dilutions. As in $1^{\text {st }}$ dilution of $70 \%$ fraction and $30 \%$ fraction no activity was observed that suggested the bio-masked entities present, had masked this activity with that particular dilution used. When it is diluted for the $2^{\text {nd }}$ time these compounds become active and started to scavenge free NO radicals. 50\% fraction had certain compounds that became active at first dilution but were masked with the $2^{\text {nd }}$ dilution as it provided with very low radical scavenging activity. Hence it was concluded that $90 \%$ fraction of Fogonia had inactive compounds while $70 \%$ and $30 \%$ fraction had significant concentration of bio-active compounds at $2^{\text {nd }}$ dilution, but $50 \%$ fraction had active compound only with $1^{\text {st }}$ dilution as compared to standard.

Biological entities in plant including polyphenols, flavonoids, tannins, terpenes, aromatic aldehydes, and alcohols but superficially polyphenols, Flavonoids are responsible activities as anti-oxidation, enzyme inhibition mechanism i.e. anti-Tyrosinase, and anti-urease activity. By above results, these activities are 
taking place with a significant differential rate in response to the chemically changing environment provided by the different fraction mixture giving a correlation among them. As reported that the total phenolic and flavonoids content provide a correlation among anti-oxidant with anti-tyrosinase, anti-urease, with reducing power of the plant [36] [37] [38] [39] [40]. Thus providing a chemically optimized environment cross-interference between the bio-entities is ensured by the successful synthesis if Ag NPs.

\section{Conclusion}

Traditional plants like Fagonia cretica are considered to be the natural, ecofriendly and less expensive source of medicine. The fractionation and double dip dilution method were used to synthesize Ag NPs and resolve the bioactive entities, responsible for synthesis. Fagonia cretica 50\% fractionation was successfully used to synthesise Ag NPs done, as a result of enhanced bio reducing activity by other probative bioactivities including anti-oxidant, anti-Tyrosinase, and anti-urease. Where $90 \%$ and $70 \%$ ethanol fraction of plant extract is more potent for Anti tyrosinase and Anti urease activity. 50\% ethanol fractions of plant extract show potential for Anti-oxidant, Anti urease. 30\% ethanol fraction of plant extract when diluted twice, it becomes stronger to show Anti-oxidant and Anti urease activity. 50\% and 30\% ethanol fraction of plant extract show Anti tyrosinase activity only when used in high concentration. Hence $50 \%$ ethanoic fraction of plant extract has more active compounds (among all fractions) involve in various pharmacological activities.

\section{Acknowledgements}

The authors would like to acknowledge the financial and technical support provided by The Islamia University of Bahawalpur, Pakistan and Higher Education Commission (HEC) funded National Research Programme for Universities (NRPU) (5194).

\section{Ethical Approval}

The study approved by Local Ethical Committee of The Islamia University Bahawalpur, Pakistan.

\section{Conflicts of Interest}

The authors have no conflict of interest.

\section{References}

[1] Hasan, M., Ullah, I., et al. (2018) Biological Entities as Chemical Reactors for Synthesis of Nanomaterials: Progress, Challenges and Future Perspective. Materials Today Chemistry, 8, 13-28. https://doi.org/10.1016/j.mtchem.2018.02.003

[2] Hasan, M., Teng, Z.Q., Iqbal, J., Awan, U., et al. (2013) Assessment of Bioreducing and Stabilizing Potential of Dragon's Blood (Dracaena Cochinchinensis, Lour. S. C. 
Chen) Resin Extract in Synthesis of Silver Nanoparticles. Nanoscience and Nanotechnology Letters, 5, 780-784. https://doi.org/10.1166/nnl.2013.1600

[3] Dang, H., Hasan, M., et al. (2014) Luteolin-Loaded Solid Lipid Nanoparticles Synthesis, Characterization, \& Improvement of Bioavailability, Pharmacokinetics in Vitro and Vivo Studies. Journal of Nanoparticle Research, 16, Article ID: 2347.

[4] Hasan, M., Iqbal, J., Awan, U., Xin, N., Dang, H., et al. (2015) LX Loaded Nanoliposomes Synthesis, Characterization and Cellular Uptake Studies in $\mathrm{H}_{2} \mathrm{O}_{2}$ Stressed SH-SY5Y Cells. Journal of Nanoscience and Nanotechnology, 15, 1320-1326.

[5] Hasan, M., Mustafa, G., Iqbal, J., Ashfaq, M. and Mahmood, N. (2018) Quantitative Proteomic Analysis of HeLa Cells in Response to Biocompatible $\mathrm{Fe}_{2} \mathrm{C} @ \mathrm{C}$ Nanoparticles: ${ }^{16} \mathrm{O} /{ }^{18} \mathrm{O}$-Labelling \& HPLC-ESI-Orbit-Trap Profiling Approach. Toxicological Research, 7, 84-92. https://doi.org/10.1039/C7TX00248C

[6] Hasan, M., et al. (2017) Biocompatibility of Iron Carbide and Detection of Metals Ions Signaling Proteomic Analysis via HPLC/ESI-Orbitrap. Nano Research, 10, 1912-1923. https://doi.org/10.1007/s12274-016-1375-4

[7] Akbar, S., et al. (2018) Raphanus Sativus Mediated Synthesis, Characterization and Biological Evaluation of Zinc Oxide Nanoparticles. Nanoscience and Nanotechnology Letters, 9, 2005-2012. https://doi.org/10.1166/nnl.2017.2550

[8] Altemimi, A., Lakhssassi, N., Baharlouei, A., Watson, D.G. and Lightfoot, D.A. (2017) Phytochemicals: Extraction, Isolation, and Identification of Bioactive Compounds from Plant Extracts. Plants, 6, 23-42. https://doi.org/10.3390/plants6040042

[9] Qureshi, H., Asif, S., Ahmed, H., Al-Kahtani, H.A. and Hayat, K. (2016) Chemical Composition and Medicinal Significance of Fagonia cretica: A Review. Natural Product Research, 30, 625-639. https://doi.org/10.1080/14786419.2015.1036268

[10] Farheen, R., Mahmood, I. and Parveen, R. (2017) Review on Medicinal and Bioactive Role of Genus Fagonia. FUUAST Journal of Biology, 7, 33-36.

[11] Naeem, K., Yawar, W., Muhammad, B. and Rehana, I. (2014) Assessment of Macronutrients and Heavy Metals in Fgoniacretica Linn of Pakistan by Atomic Spectroscopy. Bulletin of the Chemical Society of Ethiopia, 28, 177-185. https://doi.org/10.4314/bcse.v28i2.2

[12] Nazir, I., Ur Rahman, N., Alvi, Z., Hafizur Rahman, M., et al. (2017) Antidiabetic Activities of an LC/MS Fingerprinted Aqueous Extract of Fagonia cretica L. in Preclinical Models. Planta Medica, 83, 1141-1148. https://doi.org/10.1055/s-0043-107616

[13] Hussain, A., Zia, M. and Mirza, B. (2007) Cytotoxic and Antitumor Potential of Fagonia cretica L. Turkish Journal of Biology, 31, 19-24.

[14] Chopra, R., Chopra, I., et al. (1982) Indigenous Drugs of India. 2nd Edition, Academic Publishers, Calcutta.

[15] Marwat, S.K., Fazal-Ur-Rehman and Khan, I.U. (2012) Tracing the Useful Ethnophytomedicinal Recipes of Angiosperms Used against Jaundice and Hepatitis in Indo-Pak Subcontinent. World Applied Sciences Journal, 18, 1243-1252.

[16] Iqbal, P., Ahmed, D. and Asghar, M.N. (2014) A Comparative in Vitro Antioxidant Potential Profile of Extracts from Different Parts of Fagonia cretica. Asian Pacific Journal of Tropical Medicine, 7, S473-S480. https://doi.org/10.1016/S1995-7645(14)60277-7

[17] Iqbal, H., Sher, Z. and Khan, Z.U. (2011) Medicinal Plants from Salt Range Pind Dadan Khan, District Jhelum, Punjab, Pakistan. Journal of Medicinal Plants Research, 5, 2157-2168. 
[18] Badshah, L. and Hussain, F. (2011) People Preferences and Use of Local Medicinal Flora in District Tank, Pakistan. Journal of Medicinal Plants Research, 5, 22-29.

[19] Satpute, R., Bhattacharya, R., Kashyap, R.S., et al. (2012) Antioxidant Potential of Fagonia arabica against the Chemical Ischemia-Unduced in PC12 Cells. Iranian Journal of Pharmaceutical Research, 11, 303-313.

[20] Iqbal, P., Ahmed, D. and Asghar, M.N. (2014) A Comparative in Vitro Antioxidant Potential Profile of Extracts from Different Parts of Fagonia cretica. Asian Pacific Journal of Tropical Medicine, 7, S473-S480. https://doi.org/10.1016/S1995-7645(14)60277-7

[21] Rawal, A.K., Nath, D.K., Yadav, N., Pande, S., Meshram, S.U. and Biswas, S.K. (2009) Rubiacordifolia, Fagonia cretica Linn and Tinospora cordifolia Exert Anti-Inflammatory Properties by Modulating Platelet Aggregation and VEGF, COX-2 and VCAM Gene Expressions in Rat Hippocampal Slices Subjected to Ischemic Reperfusion Injury. International Journal of Applied Research in Natural Products, 2 , 19-26.

[22] Ran, Y., Wang, R., Lin, F., Hasan, M., Jia, Q., Tang, B., et al. (2014) Radio Protective Effects of Dragon's Blood and Its Extract against Gamma Irradiation in Mouse Bone Marrow Cells. Physics in Medicine, 30, 427-431.

https://doi.org/10.1016/j.ejmp.2013.12.001

[23] Deghbar, N., Mezioug, D., Kahina, T., Medjdoub, Y.M., et al. (2019) Antihydatic and Immunomodulatory Effects of Algerian Propolisethanolic Extract: In Vitro and in Vivo Study. Asian Pacific Journal of Tropical Medicine, 12, 106-116.

[24] Ran, Y., Wang, R., Gao, Q., Jia, Q., Hasan, M., Awan, M.U., et al. (2014) Dragon's Blood and Its Extracts Attenuate Radiation-Induced Oxidative Stress in Mice. Journal of Radiation Research, 55, 699-706. https://doi.org/10.1093/jrr/rru013

[25] Ran, Y., Xu, B., Wang, R., Gao, Q., Jia, Q., Hasan, M., et al. (2016) Dragon's Blood Extracts Reduce Radiation-Induced Peripheral Blood Injury and Protects Human Megakaryocyte Cells from GM-CSF Withdraw-Induced Apoptosis. Physica Medica, 32, 84-93.

[26] Mahmood, S., Fatima, T., Zulfaqar, H., Saher, R., et al. (2017) Meta-Analysis of Dragon's Blood Resin Extract as Radio-Protective Agent. Journal of Coastal Life Medicine, 5, 409-416.

[27] Touzani, S., Al-Waili, N., El Menyiy, N., Filipic, B., et al. (2018) Chemical Analysis and Antioxidant Content of Various Propolis Samples Collected from Different Regions and Their Impact on Antimicrobial Activities. Asian Pacific Journal of Tropical Medicine, 11, 436-442.

[28] Uddin, Md.S., Hossain, Md.S., Al Mamun, A., et al. (2018) Phytochemical Analysis and Antioxidant Profile of Methanolic Extract of Seed, Pulp and Peel of Baccaurea ramiflora Lour. Asian Pacific Journal of Tropical Medicine, 11, 443-450.

[29] Xin, N., Hasan, M., Li, W. and Li, Y. (2014) Juglans mandshurica Maxim Extracts Exhibit Antitumor Activity on HeLa Cells in Vitro. Molecular Medicine Reports, 9, 1313-1318. https://doi.org/10.3892/mmr.2014.1979

[30] Ozer, Ö., Berkay, M. and Bijan, K. (2007) Antityrosinase Activity of Some Plant Extracts and Formulations Containing Ellagic Acid. Pharmaceutical Biology, 45, 519-524. https://doi.org/10.1080/13880200701446746

[31] Mobley, H. and Hausinger, R. (1989) Microbial Ureases: Significance, Regulation, and Molecular Characterization. Microbiological Reviews, 53, 85-108.

[32] Iqbal, H. and Sher, Z. (2011) Medicinal Plants from Salt Range Pind Dadan Khan, District Jhelum, Punjab, Pakistan. Journal of Medicinal Plant Research, 5, 2157- 
2168.

[33] Cáceres, A., Almeda, F., et al. (2018) Anti-Urease Activity of Native Species of Genus Piper from Guatemala with Potential Application in Infection Control. International Journal of Phytocosmetics and Natural Ingredients, 5, 2. https://doi.org/10.15171/ijpni.2018.02

[34] Azmi, N., Hashim, P., Hashim, D.M., Halimoon, N. and Majid, N. (2014) Anti-Elastase, Anti-Tyrosinase and Matrix Metalloproteinase-1 Inhibitory Activity of Earthworm Extracts as Potential New Anti-Aging Agent. Asian Pacific Journal of Tropical Medicine, 4, 348-352. https://doi.org/10.12980/APJTB.4.2014C1166

[35] Baurin, N., Arnoult, E., Scior, T., Do, Q. and Bernard, P. (2002) Preliminary Screening of Some Tropical Plants for Antityrosinase Activity. Journal of Ethnopharmacology, 82, 155-158. https://doi.org/10.1016/S0378-8741(02)00174-5

[36] Lateef, M., Iqbal, L., Fatima, N., Siddiqui, K., Afza, N., et al. (2012) Evaluation of Antioxidant and Urease Imbibition Activities of Roots of Glycyrrihza glabra. Pakistan Journal of Pharmaceutical Sciences, 25, 99-102.

[37] Saadullah, M., Chaudary, B.A. and Uzair, M. (2016) Antioxidant Phytotoxic and Antiurease Activities and Total Phenolic and Flavonoid Contents of Conocorpus lancifolius. Tropical Journal of Pharmaceutical Research, 15, 555-561. https://doi.org/10.4314/tjpr.v15i3.17

[38] Nile, S.H., Keum, Y.S., Nile, A.S., Jalde, S.S. and Patel, R.V. (2017) Antioxidant Anti-Inflammatory and Enzyme Inhibitory Activity of Natural Plant Flavonoid and Their Synthesised Derivatives. Journal of Biochemical and Molecular Toxicology, 32, e22002. https://doi.org/10.1002/jbt.22002

[39] Zengin, G., Uysal, S., Ceylan, R. and Aktumsek, A. (2015) Phenolic Constituents Antioxidative and Tyrosinase Inhibitory Activity of Ornithogalum narbonense L. from Turkey: A Phytochemical Study. Industrial Crops and Products, 70, 1-6. https://doi.org/10.1016/j.indcrop.2015.03.012

[40] Athipornchai, A. and Jullapo, A. (2018) Tyrosinase Inhibitory and Antioxidant Activities of Orchid (Dendrobium spp.). South African Journal of Botany, 119, 188-192. https://doi.org/10.1016/j.sajb.2018.09.003 